\title{
Light-by-Light polarization control of 10-Gbit/s RZ and NRZ telecommunication signals
}

\author{
J. Fatome, P. Morin, S. Pitois and G. Millot
}

\begin{abstract}
Controlling the state of polarization of a light beam propagating in a standard single mode fiber by means of a lossless and instantaneous interaction is a fundamental effect of great interest for telecommunication applications and all-optical signal processing. In this work, we experimentally demonstrate light-bylight polarization control via a nonlinear interaction occurring in single mode optical fiber between a signal wave and a counterpropagating control pump beam. We observe a polarization attraction and stabilization of a 10-Gbit/s optical telecommunication signal around $1550 \mathrm{~nm}$ for either RZ or NRZ modulation format. These experimental results confirm yet another fascinating possibility to all-optical control the light properties in optical fiber.
\end{abstract}

Index Terms - Nonlinear optics, Optical Fiber, Polarization control, Signal Processing.

\section{INTRODUCTION}

I $\mathrm{N}$ many fields of photonics, especially in optical fiber based devices, the state of polarization (SOP) of light remains so far one of the most elusive uncontrolled variable, which can dramatically affect the performances of telecommunication systems. Indeed, despite the significant progress in the manufacturing process of optical fibers [1]-[2], the stochastic residual birefringence and its associated polarization mode dispersion (PMD), induces after only a few kilometers of propagation, unpredictable polarization fluctuations which can reach thousands of $\mathrm{rad} / \mathrm{s}$ [3]-[8]. Consequently, implementation of polarization sensitive signal processing such as high-contrast integrated silicon or microchip waveguides [9]-[10], nonlinear fiber based functions [11], regeneration process [12], photonic-crystal waveguides [13]-[14] are so far limited, thus delaying the development of future transparent networks. In this context, developing new functions capable to control or stabilize an arbitrarily polarized optical signal has become of a great interest in many fields of optics, first in Telecommunication systems but also in sensing, fiber laser, interferences or

Manuscript received November 14, 2010. This work was supported by the Agence Nationale de la Recherche (ANR FUTUR project: ANR-06-TCOM016) and by the Conseil Régional de Bourgogne.

J. Fatome, P. Morin, S. Pitois and G. Millot are with the Laboratoire Interdisciplinaire Carnot de Bourgogne, UMR 5209 CNRS/Université de Bourgogne, 9 av. A. Savary, 21078 Dijon, France (corresponding author to provide phone: +33 380395995; fax: +33 380395971 e-mail: jfatome@ubourgogne.fr). metrology.

Fixing the SOP of light can obviously be ensured by a standard polarizer, but in that case, the input polarization fluctuations are transformed into large intensity fluctuations at the device output, which is unacceptable in many practical situations, especially for nonlinear post-processing applications. In order to combat these impairments, a second way to stabilize the light SOP is an active polarization control based on optoelectronic elements coupled to feedback algorithms [15]-[16]. These systems have already demonstrated their efficiency but are thus limited by the electronic response time and are not fast enough to master strong polarization variations [8]. Moreover, they cannot be compatible with wavelength division multiplexing applications. On the other hand, because of their quasiinstantaneous features, nonlinear effects occurring in optical fibers have recently paid considerable attention as a possible solution to all-optical master the polarization of light. Typical and remarkable examples of such a system are the photorefractive crystal-based nonlinear polarizer reported by Heebner et al. in ref. [17] or Raman pulling by Martinelli et al. [18]-[19] as well as Brillouin effect [20]-[23] occurring in optical fibers. These different works have reported convincing experimental proofs of concept but have not yet succeed in a real in-situ Telecommunication demonstration which could stimulate future emerging applications.

Beside these works, we have identified in some previous experiments another approach of polarizing process taking place in an isotropic optical fiber pumped by two counterpropagating beams and based on a four-wave mixing phenomenon [24]. More precisely, it has been shown that a circularly polarized pump could act as a lossless polarization attractor for a signal beam propagating in the opposite direction [24]-[25]. More recently, in ref. [26], we have extended this concept to low PMD optical fibers and demonstrated the all-optical control of the SOP of a 10-Gbit/s Return-to-Zero (RZ) telecommunication signal. In this paper, we study in details this new signal processing device and experimentally demonstrate that it is possible to all-optical control and stabilize the state of polarization of 10-Gbit/s Telecommunication signals either modulated in RZ or NRZ formats. Thanks to experimental observations, we highlight the exchange of entropy occuring between signal and pump beams which originates from the polarization attraction process. We also study the influence of the pump and signal wavelength 
mismatch as well as the role of the pump spectral linewidth. Finally, in the last section, we demonstrate that our system is able to vanish strong and very fast polarization events.

\section{THEORETICAL CONSIDERATION}

The physic involved in our system relies on the nonlinear interaction based on Four Wave Mixing (FWM) occurring between two counter-propagating waves propagating in an optical fiber [24]-[25]. Indeed, it was demonstrated in previous works that two waves, a pump wave and a signal wave, injected with opposite directions in a perfectly isotropic fiber, tend to equalize their polarization ellipticities all along the fiber length whereas, in the same time, the angle between the principal axes of the pump and signal polarizations is attracted towards a value that only depends on the initial difference between the signal and pump ellipticities [24]. In this particular system, as schematically described in Fig. 1, it was theoretically and experimentally demonstrated that a pump wave injected with a circular state of polarization constitutes an attractor or funnel of that systems in such a way that the output polarization of the signal is fixed and stabilized independently of its initial SOP [24]-[25].
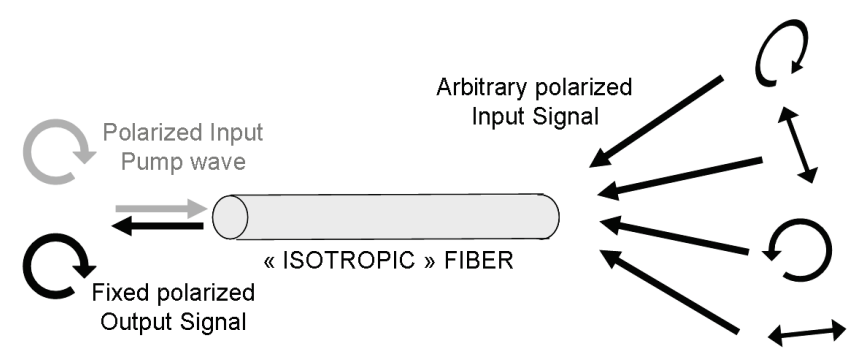

Fig. 1. Schematic illustration of the attraction process occurring in an isotropic optical fiber.

This remarkable polarization attraction effect has been experimentally observed in previous studies where it was highlighted that fiber isotropy was a key element of the experiment success, in the sense that any perturbation of this isotropy inexorably lead to a dramatic decrease of the attraction efficiency. For this reason, previous experiments have been restricted to short fiber lengths (a few meters only) with very intense nanosecond pump and signal waves (more than $50 \mathrm{~W}$ ) [27]-[28], such parameters being incompatible with telecommunications applications. In a very recent work, we have reported the experimental observation of such a phenomenon of polarization attraction in a $20-\mathrm{km}$ long standard single mode fiber [26]. In that experiment, it was then demonstrated for the first time, that it was possible to alloptical control and stabilize the SOP of a $10 \mathrm{Gbit} / \mathrm{s} \mathrm{RZ}$ optical signal. As in early-years experiments, the system was also based on the FWM occurring in optical fiber between a polarization scrambled signal and a pump beam with a fixed SOP, but the technological breakthrough was in the use of a standard low PMD optical fiber. Indeed, in such a system, the whole fiber can be considered as a concatenation of short and perfect polarization attractors as defined originally in isotropic fiber. In fact, if the local birefringence of the fiber is sufficiently low enough, one can show that a four-wave mixing (FWM) process occurs between the polarization components of the two counter-propagating waves, whatever the wavelength mismatch between the signal and pump waves [29]-[30]. More precisely, it was demonstrated that this FWM process induces a unidirectional exchange of energy between the two polarization components of the signal wave all along the fiber length. A remarkable consequence of this nonlinear interaction is that the signal polarization asymptotically converges towards a fixed value at the fiber output, independently of its initial state [29].

As illustrated in Fig. 2, because of the residual PMD and random evolution of the pump SOP, the system acts as a succession of short polarization attractors characterized by their own point of attraction. Meanwhile, as one goes along the fiber, the system adiabatically vanishes the polarization fluctuations of the input signal. Consequently, the SOP of the output signal is fixed and stabilized independently from its initial state. Moreover, because of the residual PMD and associated random walk SOP evolution along the fiber length, the system is efficient even if initial pump and signal SOPs are exactly orthogonal. Finally, it is remarkable to note that, with this configuration, the initial SOP of the pump wave can be localized everywhere on the Poincaré sphere.

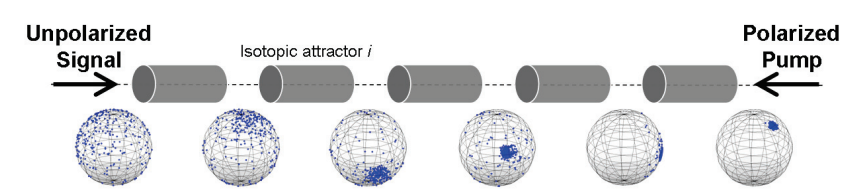

Fig. 2. Schematic illustration of the attraction process occurring in a low PMD optical fiber.

At this point, it is important to notice that the use of much longer optical fiber than in earlier experiments does not restrict anymore the polarization attraction process to only nanosecond high power experiments. The system could now be compatible with rather continuous waves, pulse trains or telecommunication signals with average powers two orders of magnitude below previous experiments obtained with quasicontinuous nanosecond pulses in a few meters of isotropic fibre [28].

\section{EXPERIMENTAL RESULTS}

\section{A. Experimental setup}

Figure 3 represents the experimental setup that we have used to observe the polarization attraction effect. The initial signal consists of a $2^{31}$-1 Return-to-Zero (RZ) or Non Returnto-Zero (NRZ) pseudo random bit sequence (PRBS) cadenced at $10 \mathrm{Gbit} / \mathrm{s}$. The central wavelength of that signal can be tuned all over the C-band. Note that the initial continuous wave was first phase modulated at a frequency of $100 \mathrm{MHz}$ in order to limit the Brillouin backscattering effect occurring within the optical fiber. A polarization scrambler was then 
inserted after the intensity modulator in order to introduce random polarization fluctuations. Before injection into the optical fiber, the signal is finally amplified by means of an Erbium doped fiber amplifier (EDFA). At the opposite end of the fiber, the counter-propagating pump beam, which acts as the attractor wave, consists of a linearly polarized incoherent wave having a spectral linewidth of $30-\mathrm{GHz}$ and a central wavelength which can be tuned all over the C-band. Note that the spectral linewidth of the pump wave was large enough to prevent any Brillouin backscattering effect within the optical fiber. The optical average power of the pump wave can be tuned between 0 and $1 \mathrm{~W}$. Two optical circulators were inserted at both ends of the fiber so as to inject and collect the pump and signal waves. The optical fiber which acts as the nonlinear Kerr medium was a $6.2-\mathrm{km}$ long Non-Zero Dispersion-Shifted Fiber (NZ-DSF) with a chromatic dispersion $\mathrm{D}=-1.6 \mathrm{ps} / \mathrm{nm} / \mathrm{km}$ at $1550 \mathrm{~nm}$ and a polarization mode dispersion (PMD) of $0.05 \mathrm{ps} / \mathrm{km}^{1 / 2}$. Note that the normal dispersion regime was carefully chosen in order to avoid any nonlinear impairment induced by modulation instability effect during the propagation, especially for the NRZ modulation format. At the system output, the signal SOP was analyzed using the usual Stokes vectors formalism and was visualized onto the Poincare sphere by means of a commercially available polarization analyzer. In order to monitor the polarization fluctuations of the signal in the time domain, we simulate the presence of a dependent polarization device by inserted an inline polarizer just before detection by a $30-\mathrm{GHz}$ bandwidth oscilloscope associated with a bit error rate (BER) or an optical sampling oscilloscope (OSO).

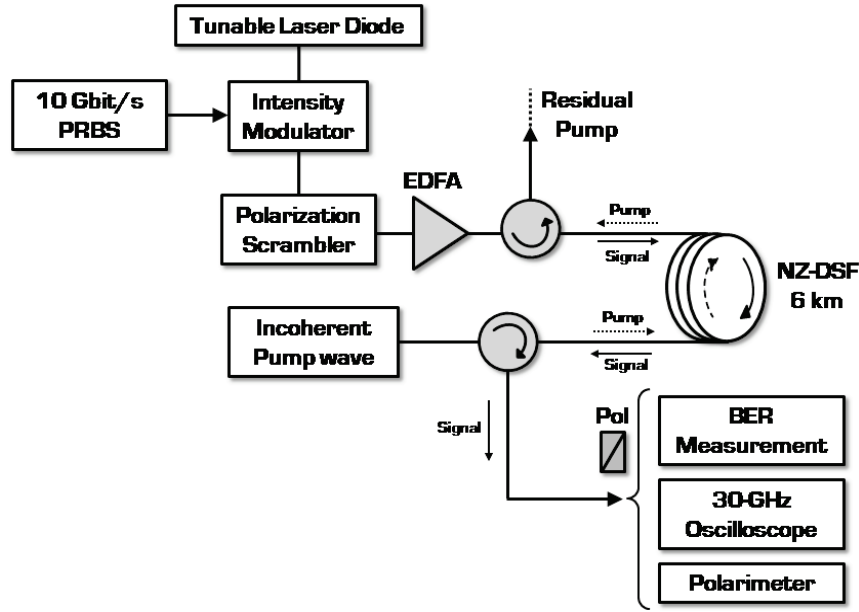

Fig. 3. Experimental setup used to observe the polarization attraction effect. PRBS : Pseudo Random Bit Sequence, EDFA : Erbium Doped Fiber Amplifier, NZ-DSF : Non-Zero Dispersion-Shifted Fiber, BER : Bit Error Rate, Pol : Linear Polarizer.

The efficiency of the polarization attraction effect was also evaluated by calculating the degree of stability (DOS) of the output signal polarization, which we define as:

$$
D O S=\sqrt{\left\langle S_{1}\right\rangle^{2}+\left\langle S_{2}\right\rangle^{2}+\left\langle S_{3}\right\rangle^{2}} / S_{0}
$$

where $\mathrm{S}_{\mathrm{i}}$ are the so-called Stokes parameters of the signal and $<>$ were evaluated over 256 initial polarizations. This definition of the DOS, close to the classical degree of polarization (DOP) but calculated on a much longer polarization fluctuation time-scale, allows to characterize the spread of the polarization fluctuations on the Poincaré sphere and thus, to quantify the efficiency of the attraction process. In fact, low values of the DOS will correspond to large temporal fluctuations of the polarization state all over the Poincaré sphere whereas a DOS value close to unity is associated with a nearly constant and stabilized polarization state.

\section{B. Polarization control of a $10 \mathrm{Gbit} / \mathrm{s}$ NRZ signal}

In a first series of experiments, we have evaluated the evolution of the DOS of a 10-Gbit/s NRZ signal at the output of the system as a function of the polarization attraction pump power. Important fluctuations of the signal polarization were introduced by means of the polarization scrambler, whereas the pump wave was injected with a fixed polarization. The results are represented in Fig. 4. The signal power was fixed to $400 \mathrm{~mW}$ while pump and signal wavelengths are $1545.4 \mathrm{~nm}$ and $1562.64 \mathrm{~nm}$, respectively. As can be seen in Fig. 4, the DOS of the signal polarization, which has initially a low value, near 0.4 , due to its initial scrambling, strongly increases when the pump power is injected into the fiber, thanks to the polarization attraction effect. At low and moderate pump power, the signal DOS increases almost linearly with respect to the pump power and finally saturates and reaches a value very close to unity for pump power above $600 \mathrm{~mW}$. Such a DOS value, close to one, is then associated to an efficient polarization attraction process and demonstrates the capability of our system to all-optical control and stabilize the SOP of a 10-Gbit/s NRZ signal.

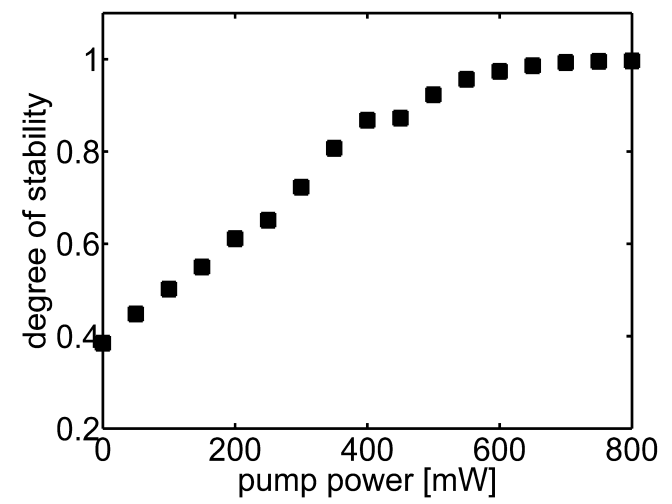

Fig. 4. Evolution of the DOS of the signal wave at the output of the fiber as a function of the pump power. The signal power was fixed to $26.3 \mathrm{dBm}(426$ $\mathrm{mW}$ ) whereas the pump power was tuned between 0 and $800 \mathrm{~mW}$.

This remarkable polarization attraction process can be more understood by directly monitor the SOP of the output signal onto the Poincaré sphere. Fig. 5 illustrates the polarization state of the 10-Gbit/s signal at the input of the system. Because of the polarization scrambling process, the points are uniformly distributed onto the sphere (Fig. 5a). When the counter-propagating pump wave is injected into the optical fiber with a $800-\mathrm{mW}$ average power, we clearly observe that most of the points are localized into a small area, indicating an attraction and stabilization of the polarization state of the 
signal wave (Fig. 5b). (a)

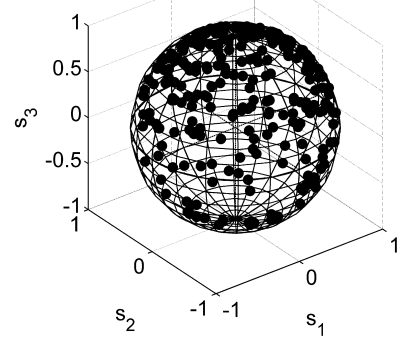

(b)

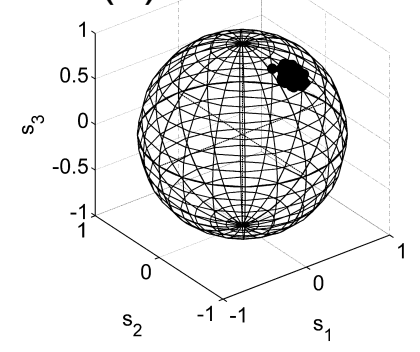

Fig. 5. State of polarization of the initially scrambled 10-Gbit/s NRZ signal plotted on the Poincaré sphere (a) At the input of the system (b) At the system output with a 400-mW signal average power and 800-mW pump power.

The efficiency of the polarization attraction process is more striking when directly monitored in the temporal domain. To this aim, we have inserted a polarization depending device, i.e. a polarizer at the output of the system ( $\mathrm{Pol}$ in Fig. 3) so as to translate the polarization fluctuations of the outcoming signal into intensity fluctuations. We have then recorded the 10 Gbit/s output signal NRZ pattern in persistent mode by means of an optical sampling oscilloscope (Fig. 6). In the pump-free configuration (Fig. 6a), the polarization fluctuations are transformed into large intensity fluctuations through the polarizer, leading to a complete dramatic loss or even extinction of the signal. By injecting the counter-propagating pump wave (Fig. 6b), a clear polarization stabilization is obtained. As can be seen, the NRZ pattern is totally recovered, all the outcoming pulses have now almost identical polarizations, so that the "one" and "zero" levels are now clearly indentified.
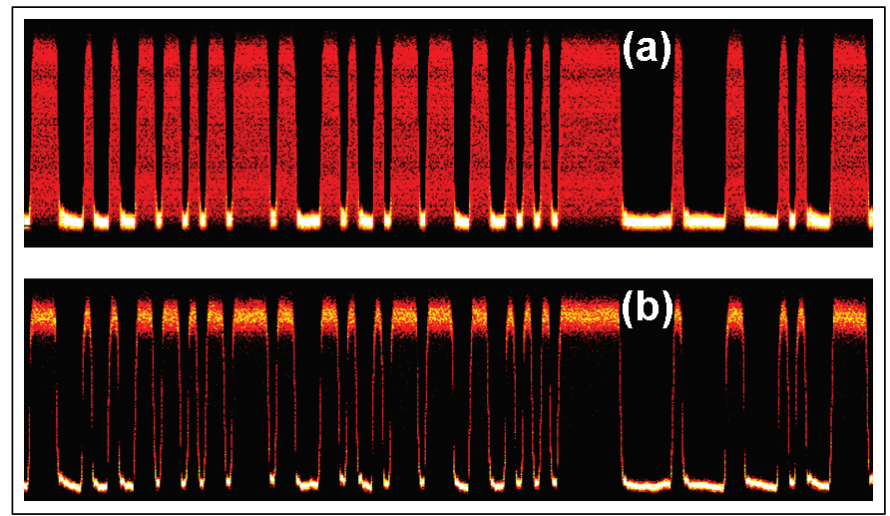

Fig. 6. Visualization of the 10-Gbit/s NRZ pattern at the system output and monitored behind a polarizer (a) without and (b) in presence of the counterpropagating pump beam.

\section{Bit error rate measurements}

Finally, we have focused our experiments on the capability of our system to improve the BER of a random bit stream carried by unpolarized NRZ signal and detected behind a polarization depend component, i.e. a polarizer.

Figure 7 represents the eye-diagrams of the polarization scrambled 10-Gbit/s NRZ signal detected at the output of the polarization attractor followed by a polarizer without (Fig. 7a) and with (Fig. 7b) injection of the counter-propagating control pump wave.

In the pump-free configuration (Fig. 7a), the initial signal polarization fluctuations are transformed into intensity fluctuations via the output polarizer, leading to a complete closure of the eye-diagram and loss of the information transmitted by the signal. By injecting the $800-\mathrm{mW}$ counterpropagating pump wave (Fig. 7b), a clear polarization stabilization is obtained. As can be seen, all the outcoming pulses have now almost identical polarizations, without additional intensity fluctuations, so that the opening of eyediagram is now efficiently recovered.

In order to underline the practical compatibility of the device with telecommunication applications, we have also measured the corresponding bit-error-rate (BER) of the NRZ $10-\mathrm{Gbit} / \mathrm{s}$ signal as a function of the average power incoming on the receiver (Fig. 7c). The reference is represented by the back-to-back configuration (i.e. at the fiber input) in circles. At the output of the system, when the polarization of the signal is scrambled and the pump off, corresponding to the eye diagram of Fig $7 \mathrm{a}$, the BER is limited to $10^{-4}$ (triangles). When the counter-propagating pump wave is applied (diamonds), the quality of the transmission is greatly improved by almost 8 orders of magnitude on the BER measurements for a $-30-\mathrm{dBm}$ average power on the receiver with negligible power penalties compared to back-to-back measurements.
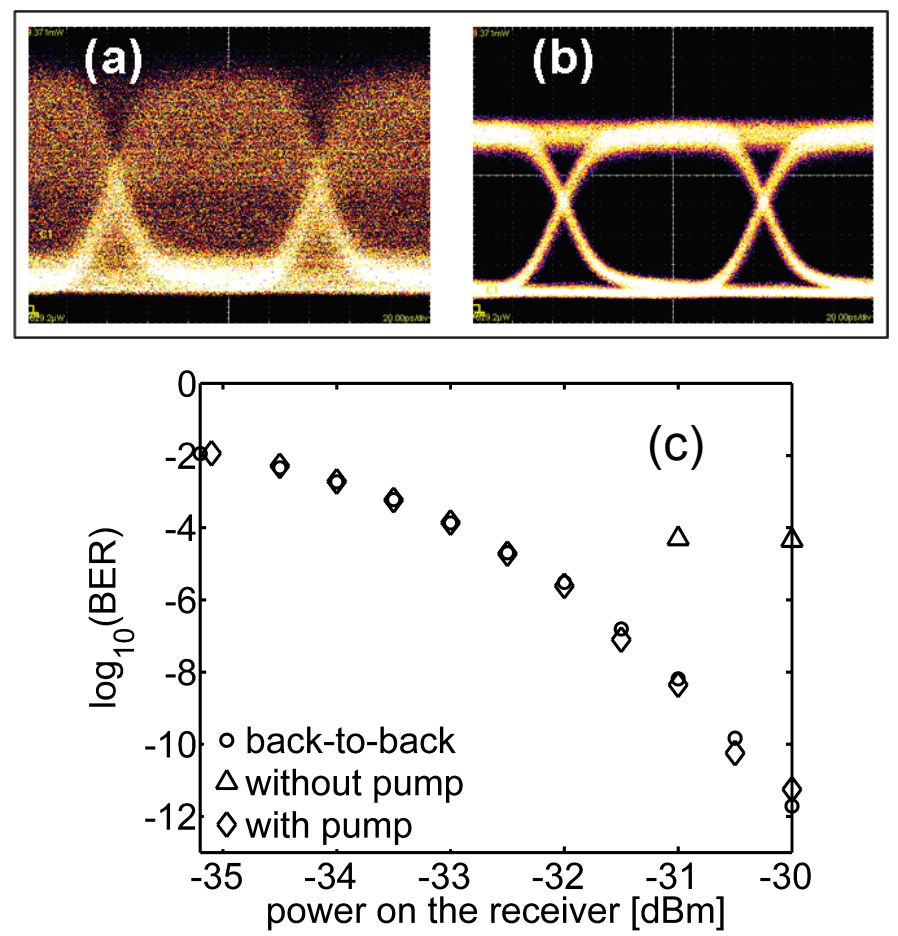

Fig. 7. (a) Eye diagram of the initially polarization scrambled 10-Gbit/s $N R Z$ signal $(400 \mathrm{~mW})$ at the output of the polarization attractor and detected behind a polarizer (a) Without counter-propagating pump wave (b) With counter-propagating pump $(800 \mathrm{~mW})$ (c) Corresponding evolution of the $B E R$ as a function of the power incoming on the receiver: back-to-back (circles), scrambled signal without (triangles) and with pump wave (diamonds). 


\section{Polarization attraction imposes entropy exchange}

Another important physical property of the polarization attractor which has to be underlined in this paper is that the total polarization entropy is supposed to be conserved during the attraction process. As a consequence, the signal polarization fluctuations are expected to be transferred to the pump polarization and finally evacuated from the system thanks to the counter-propagating configuration.

This remarkable effect is clearly visible in Fig. 8, which shows the DOS of the pump wave as a function of the signal power. In the absence of signal wave (signal power $=0 \mathrm{~mW}$ ), the pump wave polarization is not significantly affected by the propagation through the optical fiber and remains constant at fiber output, leading to a polarization degree of stability (DOS) close to unity. At the opposite, when the polarization scrambled 10-Gbit/s signal is injected at the other end of the fiber, as its average power increases, the polarization attraction effect occurs and the signal polarization fluctuations are transferred to the pump wave, leading to a spectacular decrease of its DOS.

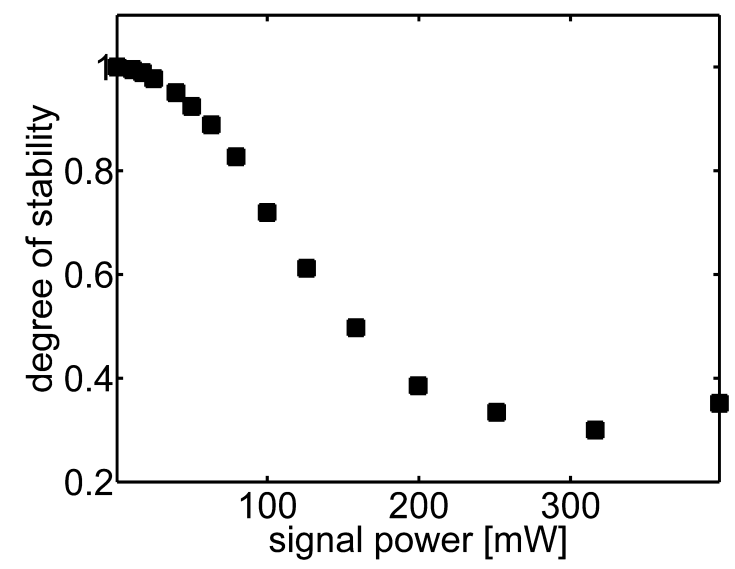

Fig. 8. Evolution of the pump DOS as a function of the 10-Gbit/s NRZ signal power, for a fixed pump power of $800 \mathrm{~mW}$ and the same pump and signal wavelengths as in Fig. 4.

This effect is also clearly visible onto the experimental Poincaré spheres depicted in Fig. 9 in which the initial fixepolarized pump wave (Fig. 9a) is completely scrambled through the polarization attraction process (Fig. 9b). (a)

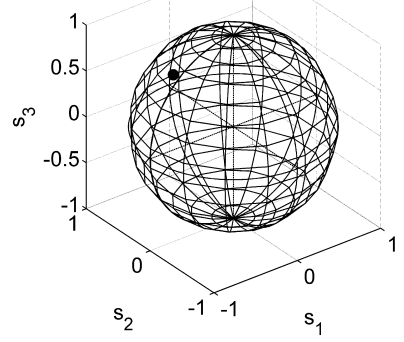

(b)

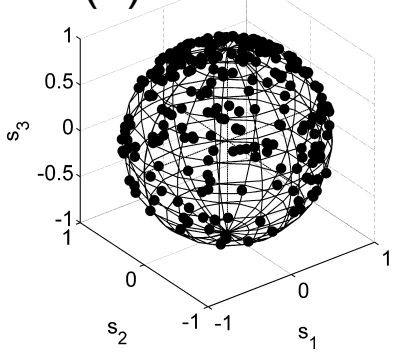

Fig. 9. State of polarization of the 800- $m W$ pump wave plotted on the Poincaré sphere (a) At the input of the system (b) At the system output with a 400-mW 10-Gbit/s NRZ signal average power.
A fascinating approach of this entropy exchange is that the attraction process allows to all-optical impose to a signal beam, a polarization trajectory onto the Poincaré sphere fixed by another. An example of this original feature is depicted in Fig. 10. An initial eight-like trajectory is imposed on the input 10-Gbit/s NRZ signal by means of the polarization scrambler (Fig. 10a) and injected into the fiber with an average power of $400 \mathrm{~mW}$. At the opposite end, the pump wave has a fixed polarization (Fig. 10b). After interaction and entropy exchange in the polarization attractor, the variations of the signal SOP are greatly reduced (Fig. 10c) while the output pump wave now describes a trajectory similar to the initial signal (Fig. 10d). This original feature of the polarization attractor could find some applications in the all-optical control of the polarization evolution, for example to impose SOP trajectories or even scrambling in sensing field or polarization depend loss test beds. (a)

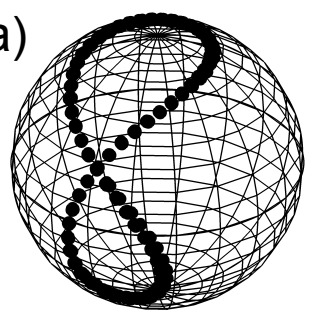

(b)

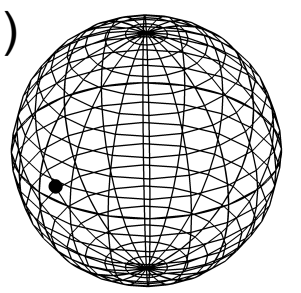

(c)
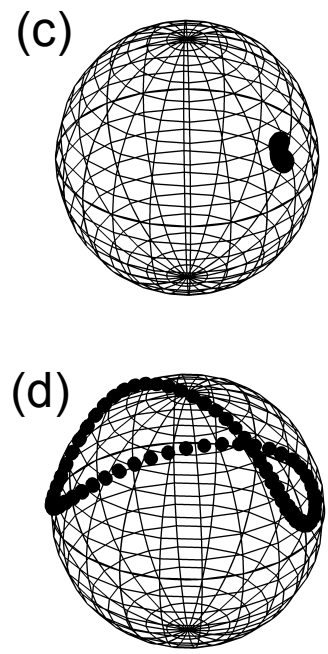

Fig. 10. State of polarization of the 400-mW 10-Gbit/s NRZ signal wave at the input of the system (a) At the output (b) State of polarization of the 800$m W$ pump wave at the input of the system (c) At the output (d).

\section{E. Influence of the pump linewidth}

In a perfectly isotropic fiber, we have shown in a previous study that the FWM process responsible for the polarization attraction process can occur whatever the degree of coherence of the counter-propagating interacting waves [31]. To quantify the robustness of this system property, we have studied the influence of the pump spectral linewidth on the polarization attraction efficiency. The results are displayed in Fig. 11, which shows the evolution of the DOS parameter as a function of pump linewidth. Signal and pump powers are $430 \mathrm{~mW}$ and $1.1 \mathrm{~W}$, respectively. Wavelengths are the same as in Fig. 4. As can be observed, no significant influence of the pump linewidth was observed in the range of $20 \mathrm{GHz}$ to $1 \mathrm{THz}$, indicating a great flexibility in the polarization attractor design. 


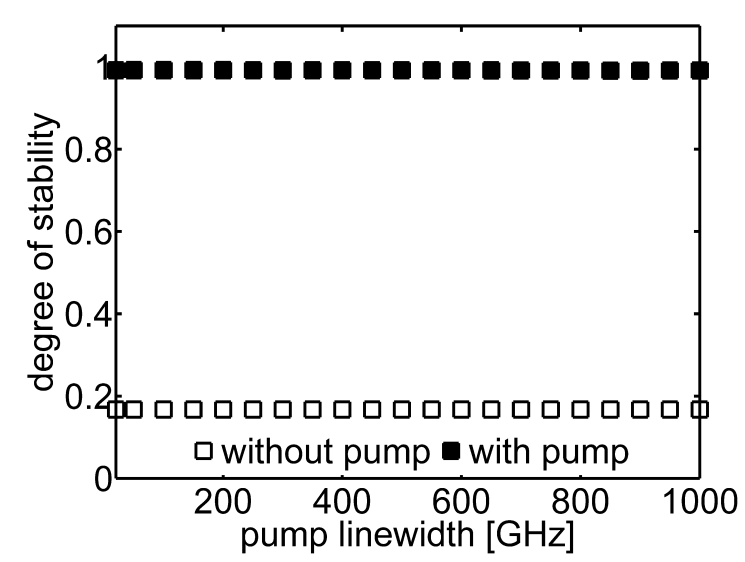

Fig. 11. DOS of the 10-Gbit/s NRZ signal wave as a function of pump linewidth for the following parameters: signal power $430 \mathrm{~mW}$, pump power $1.1 \mathrm{~W}$, wavelengths are the same as in Fig. 4 .

\section{F. Influence of the wavelength}

Finally, we have also studied the influence of a wavelength mismatch between the pump and signal waves. The experimental results are shown in Fig. 12. The pump wavelength was tuned between $1540 \mathrm{~nm}$ and $1550 \mathrm{~nm}$ whereas the signal wavelength was fixed to $1545.3 \mathrm{~nm}$. The pump and signal powers were fixed to $1.1 \mathrm{~W}$ and $700 \mathrm{~mW}$, respectively. As predicted by theory, the four wave mixing process involved in the polarization attraction effect is phase matched whatever the frequency difference between the pump and signal waves, so that the efficiency of the nonlinear interaction is not affected by the wavelength mismatch. We would like to point out that this property is of great importance for WDM applications and Raman amplification considerations.

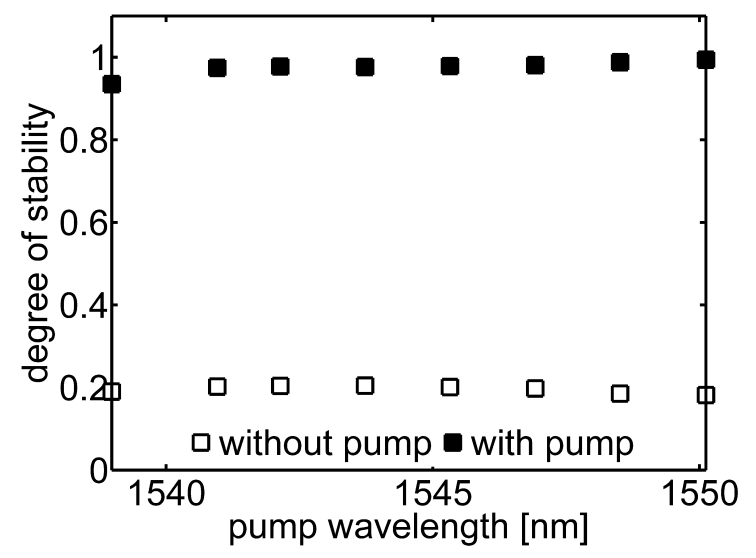

Fig. 12. Efficiency of the polarization attractor effect on a 10-Gbit/s NRZ signal as a function of the pump wavelength. The signal wavelength was fixed to $1545.3 \mathrm{~nm}$, pump and signal powers are $1.1 \mathrm{~W}$ and $700 \mathrm{~mW}$, respectively.

\section{G. $10 \mathrm{Gbit} / \mathrm{s}$ RZ results}

In ref. [26], we have had demonstrated the proof of concept of the all-optical control of the SOP on a 10-Gbit/s RZ signal. Here, we improve the quality of the device by carefully select the optical fiber involved in the attraction process. As the propagated signal undergoes a strong nonlinear regime, the sign and value of chromatic dispersion have to be designed in order to avoid dramatic impairments on the temporal profile due to the interaction of chromatic dispersion and nonlinearity. It is the reason why, compared to ref. [26], the fiber involved in these experimental results was shorter $(6 \mathrm{~km})$ and in normal dispersion regime $(\mathrm{D}=-1.6 \mathrm{ps} / \mathrm{km} . \mathrm{nm})$. The wavelength of the RZ signal was centered at $1562.6 \mathrm{~nm}$ with Gaussian-like pulses of 30 ps temporal duration and a $500-\mathrm{mW}$ average power. The wavelength of the pump beam was $1543.75 \mathrm{~nm}$ and the optimum power was find to be $1.3 \mathrm{~W}$.

The experimental results are quite similar to those described in above sections and prove that our system is transparent to the modulation format (RZ or NRZ) but rather sensitive to the input average power. Figure 13 illustrates the SOP of the 10Gbit/s signal at the input of the system (Fig. 13a) and at the output of the polarization attractor (Fig. 13b). As the SOP of the initial signal is scrambled, the points are distributed onto the whole Poincaré sphere (Fig. 13a). When the counterpropagating pump wave is injected with a $1.3-\mathrm{W}$ average power (Fig. 13b), we then clearly observe an efficient attraction process accompanied by a strong localization of the points on a small area, indicating a great stabilization of the polarization state of the signal wave.
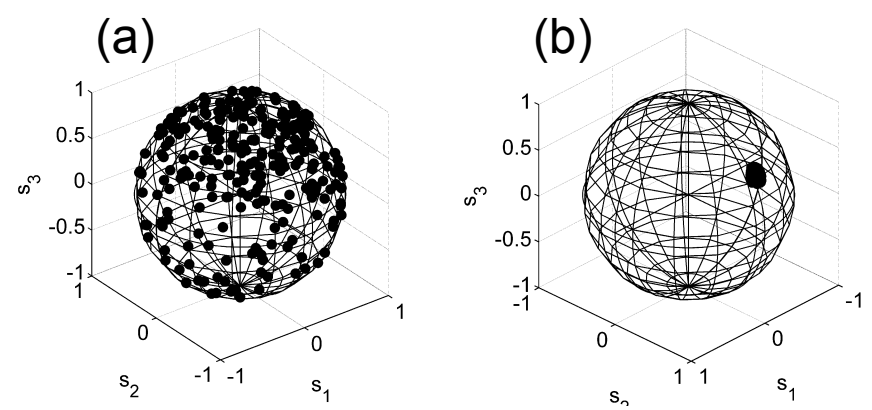

Fig. 13. State of polarization of the initially scrambled 10-Gbit/s $R Z$ signal plotted on the Poincaré sphere (a) At the input of the system (b) At the system output with a 500- $\mathrm{WW}$ signal average power and $1.3 \mathrm{~W}$ pump power.

As in the NRZ results, in order to characterize the attraction process in the temporal domain, we have then detected the resulting 10-Gbit/s RZ signal behind a polarizer. Fig. 14a shows the resulting signal when the counter-propagating pump wave is off. As the polarization is scrambled, the SOP fluctuations are transfer on the intensity profile through the polarizer resulting in large intensity fluctuations and eye closure. At the opposite, when the control pump is injected (Fig. 14b), corresponding to the polarization stabilization of Fig. $13 \mathrm{~b}$, the eye diagram is greatly recovered with negligible intensity impairments.

Finally, we have recorded the evolution of the Bit Error Rate of the polarization scrambled 10-Gbit/s RZ signal after a polarizer as a function of the power incoming on the receiver. The results are depicted in Fig. 14c, first, in back-to-back configuration and then after propagation through our device without and with the counter-propagating pump wave. In absence of control pump beam, as the polarization of the 
signal is scrambled, corresponding to the configuration of Fig $14 \mathrm{a}$, all the polarization fluctuations are transferred into intensity fluctuations through the polarizer resulting in many bit errors on the receiver. Consequently, in this configuration, the BER is thus limited to $10^{-5}$ for an average power of -30 $\mathrm{dBm}$. At the opposite, when the counter-propagating pump wave is applied, the quality of the transmission is greatly restored with negligible penalties compared to the back-toback configuration.
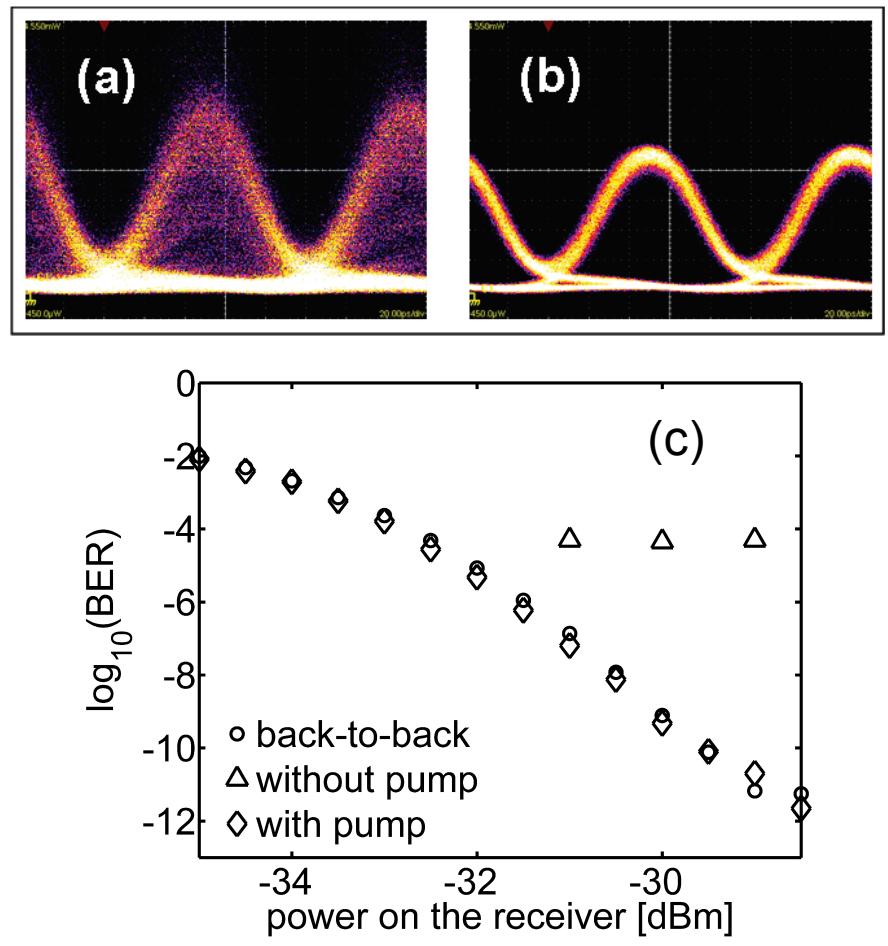

Fig. 14. (a) Eye diagram of the initially polarization scrambled 10-Gbit/s $R Z$ signal $(500 \mathrm{~mW})$ at the output of the polarization attractor and detected behind a polarizer (a) Without counter-propagating pump wave (b) With counter-propagating pump $(1.3 \mathrm{~W})$ (c) Corresponding evolution of the BER as a function of the power incoming on the receiver : back-to-back (circles), scrambled signal without (triangles) and with pump wave (diamonds).

\section{H. Burst annihilation}

In this last section, we have characterized the ability of our system to annihilate a short polarization burst, i.e. a strong and fast variation of the signal SOP [12]. Such a dramatic event is fortunately rare but could be observed in a telecommunication line and is difficult to master with present systems based on active electronic feedback [13]. To this aim, a polarization burst, having a temporal width of $6 \mathrm{~ns}$, was introduced into the initial 10-Gbit/s RZ signal by means of a $15-\mathrm{GHz}$ bandwidth optoelectronic phase modulator polarization switch.

Figure 15 shows the intensity profile of the polarization burst, monitored by means of a low bandwidth oscilloscope at the output of the system and detected behind a polarizer. In absence of counter-propagating pump beam (dashed-line), we observe the strong variation in the intensity profile, which could be disastrous for any polarization-sensitive or nonlinear processing function. When the $700-\mathrm{mW}$ counter-propagating pump beam is injected (solid line), the polarization burst was efficiently absorbed by the attraction process, leading to an error-free transmission $\left(\mathrm{BER}=10^{-12}\right)$.

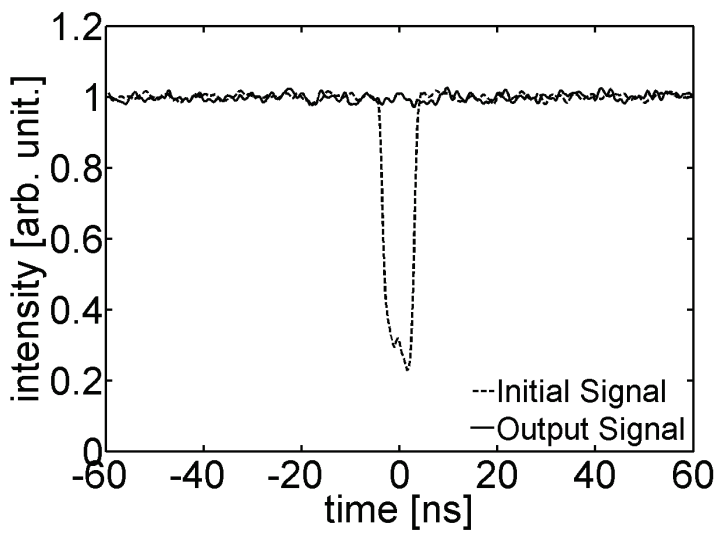

Fig. 15. Intensity profile of the 6-ns polarization burst detected behind a polarizer by means of a low bandwidth oscilloscope, without (dashed line) and with (solid line) counter-propagating pump wave.

\section{CONCLUSION}

In conclusion, we have reported the experimental demonstration of an all-optical polarization attraction process allowing control and stabilization of the state of polarization of $10-\mathrm{Gbit} / \mathrm{s}$ telecommunication signals around $1550 \mathrm{~nm}$. Our device is based on a nonlinear interaction between an incident signal beam with an arbitrary SOP and a counter-propagating control pump wave taking place in a $6-\mathrm{km}$ long single mode optical fiber characterized by low polarization mode dispersion. Based on Kerr effect, the polarization attractor is quasi instantaneous, with a large bandwidth and almost lossless. We have found that the polarization fluctuations of the 10-Gbit/s signal are vanished from the system by an exchange of entropy between the signal and pump beam. We have also show that such a device is efficient for RZ or NRZ modulation formats and that it could be tunable in the C-band. Finally, we have also demonstrated that our optical device could strongly vanish intense and fast polarization events as short as $6 \mathrm{~ns}$. Based on these observations, we think that this powerful system could find many applications in all-optical signal processing for future transparent optical networks.

\section{APPENDIX}

A short movie of the lab experiment could be find at the following URL:

http://www.youtube.com/watch?v=XC 9IvTB2bQ

\section{ACKNOWLEDGMENT}

We thank Stefano Wabnitz from the University of Brescia as well as Lucia Marazzi from PoliCom in Milan for fruitful discussions and encouragement.

\section{REFERENCES}

[1] A. J. Barlow, D. N. Payne, M. R. Hadley, and R. J. Mansfield, "Production of single-mode fibers with negligible intrinsic birefringence 
and polarization mode dispersion," Electron. Lett., vol. 17, pp. 725$726,1981$.

[2] A. Galtarossa, L. Palmieri, and A. Pizzinat, "Optimized Spinning Design for Low PMD Fibers: An Analytical Approach,” J. Lightwave Technol. Vol. 19, pp. 1502- 1512, 2001.

[3] I. P. Kaminow, and T. Li, "Optical fiber Telecommunications IV-B Systems and Impairments," 4th ed., Academic Press, San Diego, 2002.

[4] C. D. Poole, and R. E. Wagner, "Phenomenological approach to polarization dispersion in long single-mode fibers," Electron. Letters, vol. 22, pp. 1029-1030, 1986.

[5] J. P. Gordon and H. Kogelnik, "PMD fundamentals: Polarization mode dispersion in optical fibers", PNAS 97, pp. 4541-4550, 2000.

[6] J. Garnier, J. Fatome, and G. Le Meur, "Statistical analysis of pulse propagation driven by polarization-mode dispersion," J. Opt. Soc. Am. B vol. 19, pp. 1968-1977, 2002

[7] N. Gisin, B. Huttner, "Combined effects of polarization mode dispersion and polarization dependent losses in optical fibers," Opt. Commun., vol. 142, pp. 119-125, 1997

[8] M. Boroditsky, M. Brodsky, N. J. Frigo, P. Magill, and H. Rosenfeldt, "Polarization dynamics in installed fiberoptic systems" IEEE LEOS Annual Meeting Conference Proceedings (LEOS), pp. 413-414, 2005.

[9] R. Salem , M. A. Foster , A. C. Turner, D. F. Geraghty, M. Lipson, and A. L. Gaeta, "Signal regeneration using low-power four-wave mixing on silicon chip," Nature Photon., vol. 2, pp. 35-38, 2008.

[10] M. Pelusi, F. Luan, T. D. Vo, M. R. E. Lamont, S. J. Madden, D. A. Bulla, D.-Y. Choi, B. Luther-Davies, and B. J. Eggleton, "Photonicchip-based radio-frequency spectrum analyser with terahertz bandwidth," Nature Photon., vol. 3, pp. 139-143, 2009.

[11] A. Bogoni, L. Potì, P. Ghelfi, M. Scaffardi, C. Porzi, F. Ponzini, G. Meloni, G. Berrettini, A. Malacarne and G. Prati, OTDM-based optical communications networks at $160 \mathrm{Gbit} / \mathrm{s}$ and beyond, Opt. Fiber Technol., vol. 13, pp. 1-12, 2007.

[12] R. Slavík, F. Parmigiani, J. Kakande, C. Lundström, M. Sjödin, P. A. Andrekson, R. Weerasuriya, S. Sygletos, A. D. Ellis, L. Grüner-Nielsen, D. Jakobsen, S. Herstrøm, R. Phelan, J. O'Gorman, A. Bogris, D. Syvridis, S. Dasgupta, P. Petropoulos, and D. J. Richardson, "Alloptical phase and amplitude regenerator for next-generation telecommunications systems," Nature Photon., vol. 4, pp. 690-695, 2010.

[13] M. Loncar, T. Doll, J. kovi, and A. Scherer, "Design and Fabrication of Silicon Photonic Crystal Optical Waveguides," J. Lightwave Technol., Vol. 18, pp. 1402-, 1411, 2000.

[14] N. Hitoshi, Y. Sugimoto, K. Kanamoto, N. Ikeda, Y. Tanaka, Y. Nakamura, S. Ohkouchi, Y. Watanabe, K. Inoue, H. Ishikawa, and K. Asakawa, "Ultra-fast photonic crystal/quantum dot alloptical switch for future photonic networks," Opt. Express, vol. 12, pp. 6606-6614, 2004.

[15] M. Martinelli, P. Martelli, and S. M. Pietralunga, "Polarization stabilization in optical communications systems," J. Lightwave Technol., vol. 24, pp. 4172-4183, 2006.

[16] B. Koch, A. Hidayat, H. Zhang, V. Mirvoda, M. Lichtinger, D. Sandel, and R. Noè, "Optical endless polarization stabilization at $9 \mathrm{krad} / \mathrm{s}$ with FPGA-based controller," IEEE Photon. Technol. Lett., vol. 20, pp. 961963, 2008.

[17] J.E. Heebner, R.S. Bennink, R.W. Boyd and R.A. Fisher, "Conversion of unpolarized light to polarized light with greater than $50 \%$ efficiency by photorefractive two-beam coupling", Opt. Lett., vol. 25, pp. 257-259, 2000.

[18] M. Martinelli, M. Cirigliano, M. Ferrario, L. Marazzi, and P. Martelli, "Evidence of Raman-induced polarization pulling," Opt. Express, vol. 17, pp. 947-955, 2009.

[19] M. Ferrario, V. Gilardone, P. Martelli, L. Marazzi and M. Martinelli, "Effective All-Optical Polarization Control Induced by Raman Nonlinear Amplification”, in ECOC'2010, paper P1.19, 2010.

[20] L. Thevenaz, A. Zadok, A. Eyal and M. Tur, "All-optical polarization control through Brillouin amplification", in Optical Fiber Communication Conference, 2008 OSA Technical Digest CD (2008), paper OML7

[21] A. Zadok, E. Zilka, A. Eyal, L. Thévenaz, and M. Tur, "Vector analysis of stimulated Brillouin scattering amplification in standard single-mode fibers," Opt. Express , vol. 16, pp. 21692-21707, 2008.
[22] J. Fatome, S. Pitois, and G. Millot, "Experimental evidence of Brillouininduced polarization wheeling in highly birefringent optical fibers," Opt. Express, vol. 17, pp. 12612-12618, 2009.

[23] M. Sagues and A. Loayssa, "Orthogonally polarized optical single sideband modulation for microwave photonics processing using stimulated Brillouin scattering," Opt. Express, vol. 18, pp. 2290622914, 2010.

[24] S. Pitois, G. Millot, and S. Wabnitz, "Nonlinear polarization dynamics of counterpropagating waves in an isotropic optical fiber: theory and experiments," J. Opt. Soc. Am. B, vol. 18, pp. 432-443, 2001

[25] S. Pitois, A. Picozzi, G. Millot, H.R. Jauslin and M. Haelterman, "Polarization and modal attractors in conservative counterpropagating four-wave interaction", Europhys. Lett., vol. 70, pp. 88-94, 2005.

[26] J. Fatome, S. Pitois, P. Morin, and G. Millot, "Observation of light-bylight polarization control and stabilization in optical fibre for telecommunication applications," Opt. Express, vol. 18, pp. 1531115317,2010

[27] S. Pitois, A. Sauter and G. Millot, "Simultaneous achievement of polarization attraction and Raman amplification in isotropic optical fibers", Opt. Lett., vol. 29, pp. 599-601, 2004

[28] S. Pitois, J. Fatome, and G. Millot, "Polarization attraction using counter-propagating waves in optical fiber at telecommunication wavelengths," Opt. Express, vol. 16, pp. 6646-6651, 2008.

[29] V. V. Kozlov, J. Nuno, and S. Wabnitz, "Theory of lossless polarization attraction in telecommunication fibers,", J. Opt. Soc. Am. B, vol. 28, No. 1,2011

[30] V. V. Kozlov, and S. Wabnitz, "Theoretical study of polarization attraction in high birefringence and spun fibers," Opt. Lett., vol. 35, No.22, November 15,2010

[31] A. Picozzi, M. Haelterman, S. Pitois, and G. Millot, "Incoherent Solitons in Instantaneous Response Nonlinear Media," Phys. Rev. Lett., vol. 92, 143906, 2004.

Julien Fatome was born in Charleville-Méziéres, France in 1978. After graduating from the engineering school ESIREM, Dijon, France, he received the DEA degree in 2000 and the Ph.D. degree in physics for studies of ultrashort pulse propagation at $160-\mathrm{Gb} / \mathrm{s}$ in dispersion managed optical fiber lines in 2004, both from the University of Bourgogne, Dijon, France. In 2005, he became a Research Engineer in the Centre National de la Recherche Scientifique (CNRS), Laboratoire Interdisciplinaire Carnot de Bourgogne, University of Bourgogne. He is currently carrying out research in nonlinear effects, pulse trains generation at ultrahigh bit rate as well as all-optical nonlinear processing and polarization control. He has published over 70 contributions in journals and conference proceedings.

Philippe Morin was born in Epinal, France, in 1982. He received the Master degree from the University of Burgundy, France, in 2009. He is currently $\mathrm{Ph} . \mathrm{D}$ student at the Laboratoire Interdisciplinaire Carnot de Bourgogne and carrying out its research on the all-optical control of the polarization state of light and especially, on the polarization attraction process in optical fiber.

Stéphane Pitois was born in Beaune, France, in 1974. He received the Ph.D. degree in physics for studies of modulational instability and domain walls in optical fibers from the University of Burgundy, Dijon, France in 2000 and then carried out postdoctoral research with the Department of Optics and Acoustics, Free University of Brussels, Belgium, on nonlinear effects in dynamical Bragg gratings. In 2001, he became a Researcher at the Centre National de la Recherche Scientifique (CNRS), Department of Physics, University of Burgundy. He has published over 100 contributions in journals, books, and conference proceedings. His research interests include nonlinear effects and pulse trains propagation in optical fibers

Guy Millot was born in Alligny-en-Morvan, France, in 1960. He received the $\mathrm{Ph} . \mathrm{D}$. degree on laser Raman spectroscopy in gases from the University of Burgundy, Dijon, France, in 1986. Since 1994, he is a Full Professor with the Physics Department, University of Burgundy. His research interests have been focused on nonlinear effects in optical fibers, modulational instabilities, solitons, generation, propagation and characterization of optical pulse trains at high repetition rates, stimulated Raman scattering, frequency conversion, and applications in optical communications. He has published over 200 contributions in journals, books, and conference proceedings. 